\title{
New physics searches with the International Large Detector at the ILC
}

\author{
Mikael Berggren ${ }^{a, \dagger, *}$ \\ ${ }^{a}$ Deutsches Elektronen-Synchrotron DESY, \\ Notkestr. 85, 22607 Hamburg, Germany \\ E-mail: mikael.berggren@desy.de
}

Although the LHC experiments have searched for and excluded many proposed new particles up to masses close to $1 \mathrm{TeV}$, there are many scenarios that are difficult to address at a hadron collider. This talk will review a number of these scenarios and present the expectations for searches at an electron-positron collider such as the International Linear Collider. The cases discussed include the light Higgsino, the $\tilde{\tau}$ slepton in the coannihilation region relevant to dark matter, as well as other SUSY signatures. The studies are based on the ILD concept at the ILC.

*** The European Physical Society Conference on High Energy Physics (EPS-HEP2021), ***

*** 26-30 July $2021 * * *$

*** Online conference, jointly organized by Universität Hamburg and the research center DESY ***

\footnotetext{
*Speaker

$\dagger$ On behalf of the ILD concept group
} 


\section{Introduction: ILC and ILD and their strong points for searches}

The International Linear Collider (the ILC, Fig. 1) will collide polarised electrons with polarised positrons. Centre-of-mass energies will range from $250 \mathrm{GeV}$ to $500 \mathrm{GeV}$, with an upgrade path to $1 \mathrm{TeV}$ defined. An $E_{C M S}=M_{Z}$ option is also foreseeable. As the $e^{+} e^{-}$initial state implies electroweak production, the background rates will be quite low. This has consequences for the detector design and optimisation: The detectors can feature close to $4 \pi$ coverage, and they do not need to be radiation hard, so that the tracking system in front of calorimeters can have a thickness as low as a few percent of a radiation-length. In addition, the low rates means that the detectors needn't be triggered, so that all produced events will be available to analysis. Furthermore, at an $e^{+} e^{-}$machine, point-like objects are brought into collision, meaning that the initial state is fully known.

The ILC [1] has a defined 20 year running scenario, yielding integrated luminosities of 2 and $4 \mathrm{ab}^{-1}$ at $E_{C M S}=250$ and $500 \mathrm{GeV}$, respectively, and could deliver $8 \mathrm{ab}^{-1}$ at the possible upgrade to $1 \mathrm{TeV}$. To construct the ILC is currently under high-level political consideration in Japan.
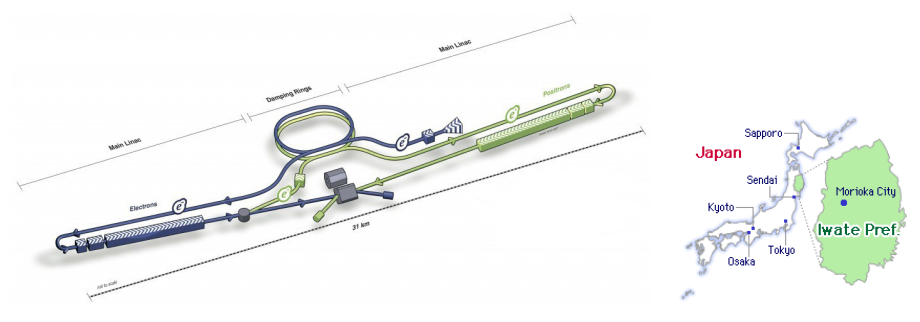

Figure 1: Schematic of the ILC and the location of the proposed site in Japan's Tohoko region.

The excellent conditions provided by the accelerator need to be matched by excellent performance of the detectors. Both precision SM measurements as well as Beyond the Standard Model (BSM) searches or measurements will require a jet energy resolution of 3-4\%, an asymptotic momentum resolution of $\sigma\left(1 / p_{\perp}\right)=2 \times 10^{-5} \mathrm{GeV}^{-1}$, and measurement of impact-parameters better than $5 \mu$. In addition, powerful particle identification capabilities are needed. The detector should be hermetic, with the only gaps in acceptance being the unavoidable vacuum pipes bringing the beams into the detector. Furthermore, the system should be capable to register data without any trigger.

In the ILD concept [2], illustrated in Fig. 2, a low mass, high precision, tracker with PID capabilities is achieved by having a Time Projection Chamber as the main tracker, enhanced by silicon trackers both inside and outside the

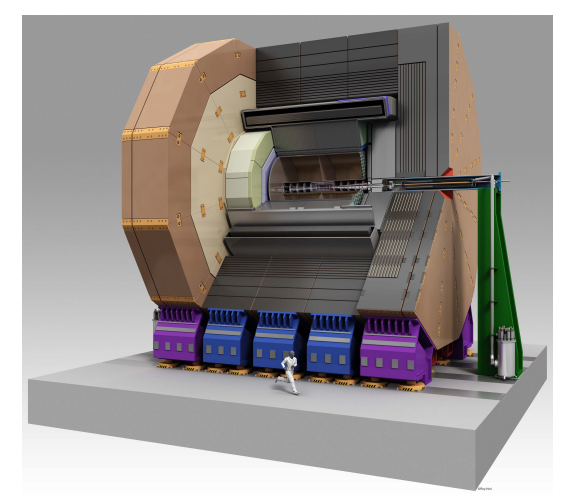

Figure 2: Artist's view of the ILD concept 
TPC. To achieve the needed jet energy resolution, ILD incorporates high granularity calorimeters optimised for particle flow. The entire system can be operated in power-pulsing mode, i.e. with the electronics being switched off between bunch-trains. In this mode, no active cooling will be needed.

\section{BSM at ILC: The SUSY case}

In this short contribution, we concentrate on one theory for physics beyond the standard model, namely SUSY[3]. Not only is SUSY the most complete theory of BSM, it can also serves as a boiler-plate for BSM in general, since almost any new topology can be obtained in some flavour of SUSY, in particular if also possible violation of R-parity and/or CP-symmetry, or non-minimal models are considered. In addition, it is the paradigm that has been most studied with detailed detector simulation. In most cases, studies were done with full simulation of the ILD, with all SM backgrounds, and all beam-induced backgrounds included. It is true that SUSY is under some stress by recent LHC results. However, ILC offers different angles to explore the properties of SUSY compared to LHC, e.g. loop-hole free searches, and complete coverage of compressed spectra.

Naturalness, the hierarchy problem, the nature of dark matter (DM), or the observed value of the magnetic moment of the muon, all prefer a light electroweak sector of SUSY. Except for the third generation squarks, the coloured sector - where pp machines excel - does not provide any insight into any of these issues. In addition, many models point into this direction: If the Lightest SUSY Particle (the LSP) is Higgsino or Wino, there must be other bosinos close in mass to the LSP, since the $\tilde{H}$ and $\tilde{W}$ fields have several components, leading to a close relation between the physical bosino states; only a Bino-LSP can have large difference, $\Delta(M)$, between the LSP and the Next to Lightest SUSY Particle (the NLSP). Furthermore, if the LSP is Higgsino, one can obtain Natural SUSY: In such models one finds that requiring low fine-tuning leads to the condition that the Higgsino mass-parameter $\mu$ must be $O\left(m_{Z}\right)$, i.e. an LSP at the weak scale. In the case of such compressed, low $\Delta(M)$, spectra, most sparticle-decays are via cascades, where the last decay in the cascade - that to SM particles and the LSP - features small $\Delta(M)$. For such decays, current LHC limits are for specific models, and only the limits from LEPII are model-independent. In fact, current observations from LHC13, LEP, g-2, DM (assumed to be 100\% LSP), and precision observables taken together also point to a compressed spectrum [4].

\subsection{SUSY with no loop-holes}

At ILC one can perform a loophole free search for SUSY because in SUSY, the properties of NLSP production and decay are completely predicted for given LSP and NLSP masses, due to SUSY-principle: "sparticles couples as particles". Note that this does not depend on the (model dependent) SUSY breaking mechanism. By definition, there is only one NLSP, and it must have $100 \%$ BR to its (on- or off-shell) SM-partner and the (stable or unstable) LSP. Also, there is only a handful of possible candidates to be the NLSP. Hence by performing searches for every possible NLSP, model independent exclusion and discovery reaches in the $M_{N L S P}-M_{L S P}$ plane, separately for each NLSP candidate, or globally, by determining which NLSP gives the weakest limit at any point. There will be no loopholes to the conclusion. Examples of this procedure are shown in Fig. 3 for the cases of a $\tilde{\chi}_{1}^{ \pm}[5]$ or a $\tilde{\tau}_{1}$ [6] NLSP. The $\tilde{\chi}_{1}^{ \pm}$is a conservative extrapolation from the 

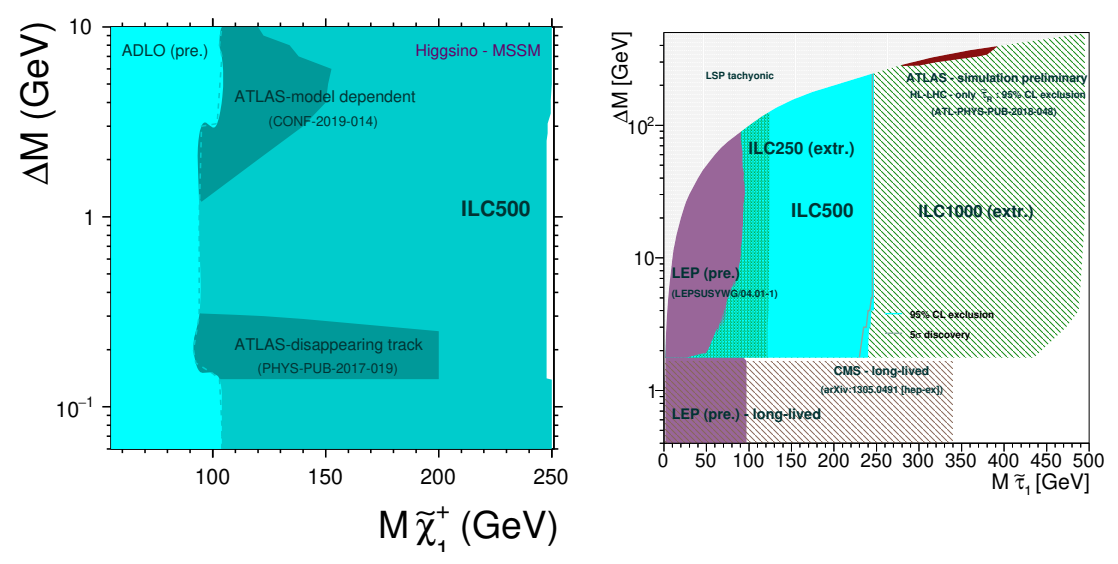

Figure 3: Exclusion and discovery reaches for a $\tilde{\chi}_{1}^{ \pm}$(left), or a $\tilde{\tau}_{1}$ (right).

LEP results, while the $\tilde{\tau}_{1}$ one is obtained with detailed fast simulation of ILD, where the $\tilde{\tau}$ and LSP properties were chosen such that the limit is the weakest possible one, i.e. the experimentally "worst possible" case. In the figure, it can be seen that the discovery and exclusion reaches are almost the same, and reach quite close to the kinematic limit $2 M_{N L S P}=E_{C M S}$. It should also be noted that the HL-LHC projection from ATLAS is exclusion only, and is for specific assumptions on the $\tilde{\tau}$ properties, assumptions that are not the most pessimistic. In Fig. 4, the various current or projected limits are shown in a single plot $[5,7]$. It should be noted that below the heavy black line, GUT unification of the Bino and Wino mass-parameters $M_{1}$ and $M_{2}$ is not possible: The difference between $M_{\tilde{\chi}_{1}^{0}}$ and $M_{\tilde{\chi}_{1}^{ \pm}}$cannot be larger than what the line indicates, if such a unification is realised.

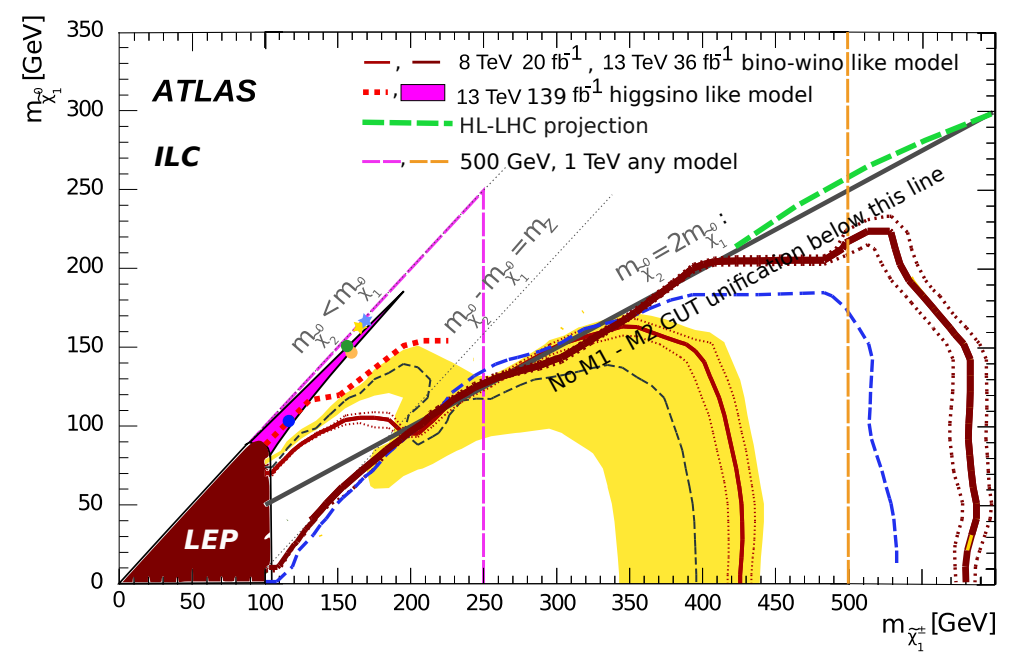

Figure 4: Observed or projected exclusion regions for $\tilde{\chi}_{1}^{ \pm}$NLSP, for LEPII, LHC, HL-LHC and for ILC-500 and ILD-1000 The symbols indicate where the higgsino LSP models shown in Fig. 6 are located. 


\subsection{SUSY at ILC: discovery in a week, then precision measurements.}

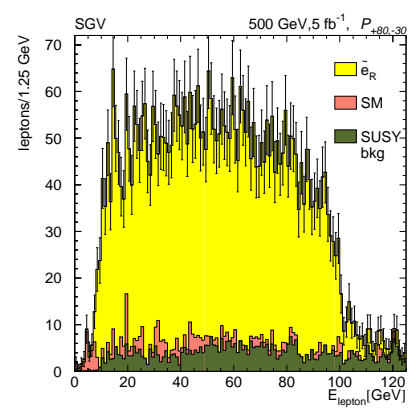

Figure 5: The ẽ signal after $5 \mathrm{fb}^{-1}$ have been collected.
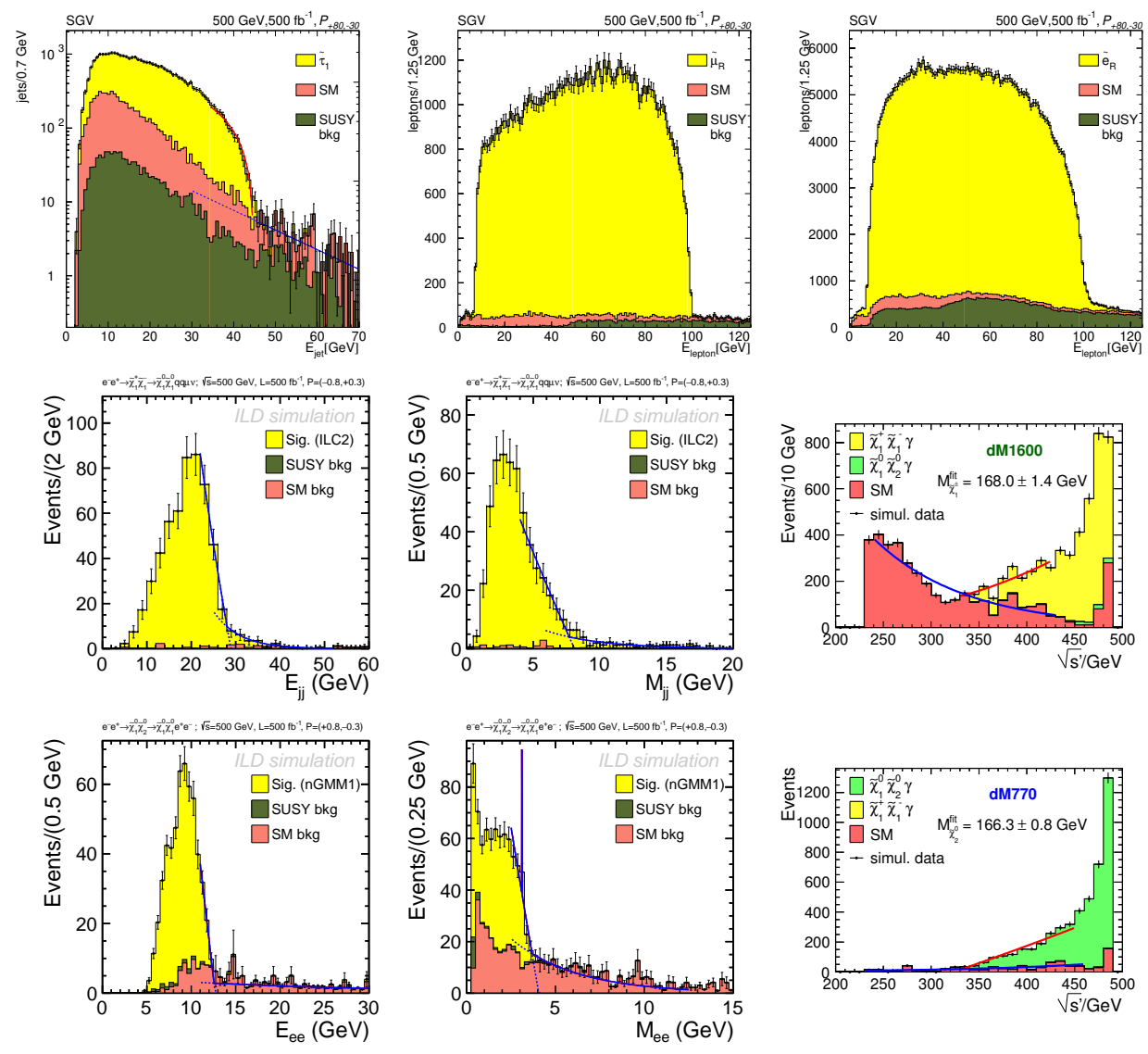

Figure 6: Top row: $\tilde{\tau}, \tilde{\mu}$ and ẽ spectra. Middle and bottom rows: Observables for three different HiggsinoLSP models. The middle row shows the case of $\tilde{\chi}_{1}^{ \pm}$production, the bottom one that of $\tilde{\chi}_{2}^{0}$ production. 
would be expected, obtained by ILD detector simulation studies: Typical slepton signal $(\tilde{\tau}, \tilde{\mu}$ and $\tilde{e})$ in the top row, in a $\tilde{\tau}$ co-annihilation model (FastSim) [8]. Typical chargino and neutralino signals in different Higgsino LSP models are shown in the following rows. The left-hand two plots are models with moderate (a few to some tens $\mathrm{GeV}$ ) $\Delta M$ (FullSim) [9], while the right-hand ones is for a model with very low (sub-GeV) $\Delta M$ (Fast/FullSim) [10]. In all the illustrated cases, it was found that the SUSY masses could be determined at the sub-percent level, the polarised production cross-sections to the level of a few percent. Many other properties could also be obtained from the same data, such as decay branching fractions, mixing angles, and sparticle spin.

\section{Conclusions}

Sometimes, the capabilities for the direct discovery of new particles at the ILC exceed those of the LHC, since ILC provides a well-defined initial state, and a clean environment without QCD backgrounds. ILC also is extendable in energy and features polarised beams. In addition, detectors like ILD, will be factors more precise, will be hermetic, and will not need for to be triggered.

Many ILC - LHC synergies are expected, from the high energy-reach of LHC versus the high sensitivity of the ILC. In particular, for SUSY, the high mass reach of LHC is ideally complemented by the sensitivity for low $\Delta(M)$ at ILC. If SUSY is reachable at the ILC, it means $5 \sigma$ discovery, and precision measurements. This input might be just what is needed for LHC to transform a $3 \sigma$ excess to a discovery of states beyond the reach of ILC.

\section{References}

[1] C. Adolphsen, M. Barone, B. Barish, et al., [arXiv:1306.6328 [physics.acc-ph]].

[2] H. Abramowicz et al. [ILD Concept Group], [arXiv:2003.01116 [physics.ins-det]].

[3] J. Wess and B. Zumino, Nucl. Phys. B 70 (1974), 39-50.

[4] E. Bagnaschi, et al. Eur. Phys. J. C 78 (2018) no.3, 256 [arXiv:1710.11091 [hep-ph]].

[5] M. T. Núñez Pardo de Vera, M. Berggren and J. List, [arXiv:2002.01239 [hep-ph]].

[6] M. T. Núñez Pardo de Vera, M. Berggren and J. List, [arXiv:2105.08616 [hep-ph]].

[7] M. Aaboud et al. [ATLAS], Eur. Phys. J. C 78 (2018) no.12, 995 [arXiv: 1803.02762 [hep-ex]]; G. Aad et al. [ATLAS], Phys. Rev. D 101 (2020) no.5, 052005 [arXiv:1911.12606 [hep-ex]]; G. Aad et al. [ATLAS], [arXiv:2106.01676 [hep-ex]]; [ATLAS], ATL-PHYS-PUB-2018-048; LEP LEPSUSYWG/02-04.1.

[8] M. Berggren, et al. Eur. Phys. J. C 76 (2016) no.4, 183 [arXiv:1508.04383 [hep-ph]].

[9] H. Baer, et al. Phys. Rev. D 101 (2020) no.9, 095026 [arXiv:1912.06643 [hep-ex]].

[10] H. Sert, et al. Eur. Phys. J. C 73 (2013) no.12, 2660 [arXiv:1307.3566 [hep-ph]]. 